\title{
Mô Phỏng Quá Trình Phục Vụ Ngırời Đọc Ơ Một Thư Viện
}

\author{
Doàn Phan Tân \\ Khoa thư viẹn \\ Trường đại học Văı hóa Hà nội
}

\section{Mô hình toán học của quá trình phục vụ người đọc ở một thư viện}

Quá trỉnh đến của một yêu càu của người đọc tại mọt thưviện là quá trình ngẫu nhiên. Giả sử ký hiẹu $T_{n}$ là thời đièm đến của yeu cầu thứ $n$ sau thời điểm ban đầu $T_{0}=0$.

Khoảng thờ gian

$$
\tau_{n}=T_{n}-T_{n-1}, n=1,2, \ldots
$$

gọi là khoảng thời gian giữa hai ycu câu liên tiếp. Ta nhận thấy rằng các khoảng thờ gian này là những biến ngẫu nhien độc lạp, không am, tuân theo cùng một quy luật xác suất nào đó. Ta gọi đó là quá trính đến của dòng yêu câu.

Giả sử phòng đọc đọc của thư viện có một kênh phục vụ. Néu tại thời điểm người đọc đưa yêu câu kiuh phục vụ rõi, thî yèu cầu được đáp ứıg ngay, còn nếu kênh phục vụ bận thì người đọc phài xêp hàng đợi. Ta luân già thiết rằng người dọc được phục vụ theo thử tự dển, không có uru tiên.

Thờ gian cần thiết dể phục vụ một yêu cầu gọi là thời gian phục vụ. Ta ký hiệu $t_{n}$ là thời gian phục vụ người đọc thứ $n, n=1,2, \ldots$. Dó cũng là nhũng biến ngẫu nhiên độc lập, không âm tuân theo một quy luật xắc suát nào đó. Khoảng thời gian giữa thời điềm đến của một yêu càu và thời điền yêu cảu bắt dàu được plụ̣c vụ gọi là thời gian đợi. Thời gian đợi của người đọc thứ $n$ ký hị̣u là $u_{n}$. Néu khòng còn người nào trı̛ớc thời điểm đến $T_{n}$ chưa được phục vụ thi $w_{n}=0$.

Nếu gọi $R_{n}$ là thời điêm người dọc thứ $n$ nhận được tài liệu và rời hệ thì

$$
R_{n}=T_{n}+w_{n}+t_{n}, n=1,2, \ldots
$$


Thời gian đợi của người đọc thứ $n$ rõ ràng bằng 0 nếu anh ta đưa yêu cầu sau lúc người đọc thứ $n-1$ rời hệ tại thời điểm $R_{n-1}$. Vì thể ta có

$w_{n}= \begin{cases}o, & \text { nếu } R_{n-1} \leq T_{n} \\ R_{n-1}-T_{n} & \text { nếu } R_{n-1}>T_{n} .\end{cases}$

Tổng thời gian đợi và thời gian phục vụ một yêu cầu gọi là thời gian lưu của người đọc tren hệ. Ký hiệu $d_{n}$ là thời gian lưu của người đọc thư $n$ ta có

$$
d_{n}=w_{n}+t_{n}
$$

Ta thấy rằng thời gian đợi cưa người đọc thứ $n$ cũng bằng 0 nếu khoảng thời gian giữa anh ta và người đến trước là $\tau_{n}$ không nhỏ hơn khoảng thời gian $d_{n-1}$ mà người đến trước lưu trên hệ. Vì vậy ta có

$$
w_{n}=\left\{\begin{array}{ll}
0 & \text { nếu } d_{n-1} \leq \tau_{n}, \\
d_{n-1}-\tau_{n} & \text { nếu } d_{n-1}>\tau_{n}
\end{array} .\right.
$$

Ta cũng nhận thấy nếu người đọc thứ $n-1$ rời hệ mà người đọc thứ $n$ chưa tới thì hệ rỗi. Ký hiệu thời gian rỗi này là $f_{n}$ thì ta có

$$
\begin{gathered}
\qquad f_{n}= \begin{cases}T_{n}-R_{n} & \text { nếu } T_{n}>R_{n-1} \\
0 & \text { nếu } T_{n} \leq R_{n-1}\end{cases} \\
= \begin{cases}\tau_{n}-d_{n-1} & \text { nếu } \tau_{n}>R_{n-1} \\
0 & \text { nếu } \tau_{n} \leq d_{n-1} .\end{cases}
\end{gathered}
$$

Khoảng thời gian mà hệ liên tục bận gọi là thời kỳ bận. Trong thời kỳ bận có thể có một số người đọc có mặt ở hệ đợi hoặc đang được phục vụ. Ta ký hiệu số người đọc có mặt trên hệ ở thời diêm $t$ là $x_{t}$. Khi đó $x_{t}=0$ có nghĩa là hệ rỗi tại thời điểm $t$, còn $x_{t}=n$ có nghía là tại thời điềm $t$ hệ đang bận và có $n-1$ người đọc đang đợi được phục vụ.

Ta ký hiệu $v_{t}$ là tổng thời gian phục vụ $x_{t}$ người đọc tại thời điểm $t$ thì ta có

$v_{t}= \begin{cases}0 & \text { nếu } x_{t}=0 \\ t_{1}^{\prime}+t_{2}+\ldots+t_{n} & \text { nếu } x_{t}=n \geq 1 .\end{cases}$

trong đó $t_{1}^{\prime}$ là thời gian phục vụ còn lại của người đọc đang được phục vụ tại thời điểm $t$, còn $t_{2}, \ldots, t_{n}$ là thời gian phục vụ của $n-1$ người đọc tại thời điểm $t$.

Biều diễn đồ thị của $v_{t}$ và $x_{t}$ được trình bày ở hỉnh vẽ dưới đây cho ta hình ảnh minh họa các khái niệm trên.

Các yếu tổ chính tác động lên quá trỉnh phục vụ người đọc ở một thư viện là:

- Quy luật đển của dòng yêu cẩu của người đọc.

- Quy luật về thợi gian ph ục vụ một yêu cầu.

- Quy luật về thời gian đọi của người đọc. 

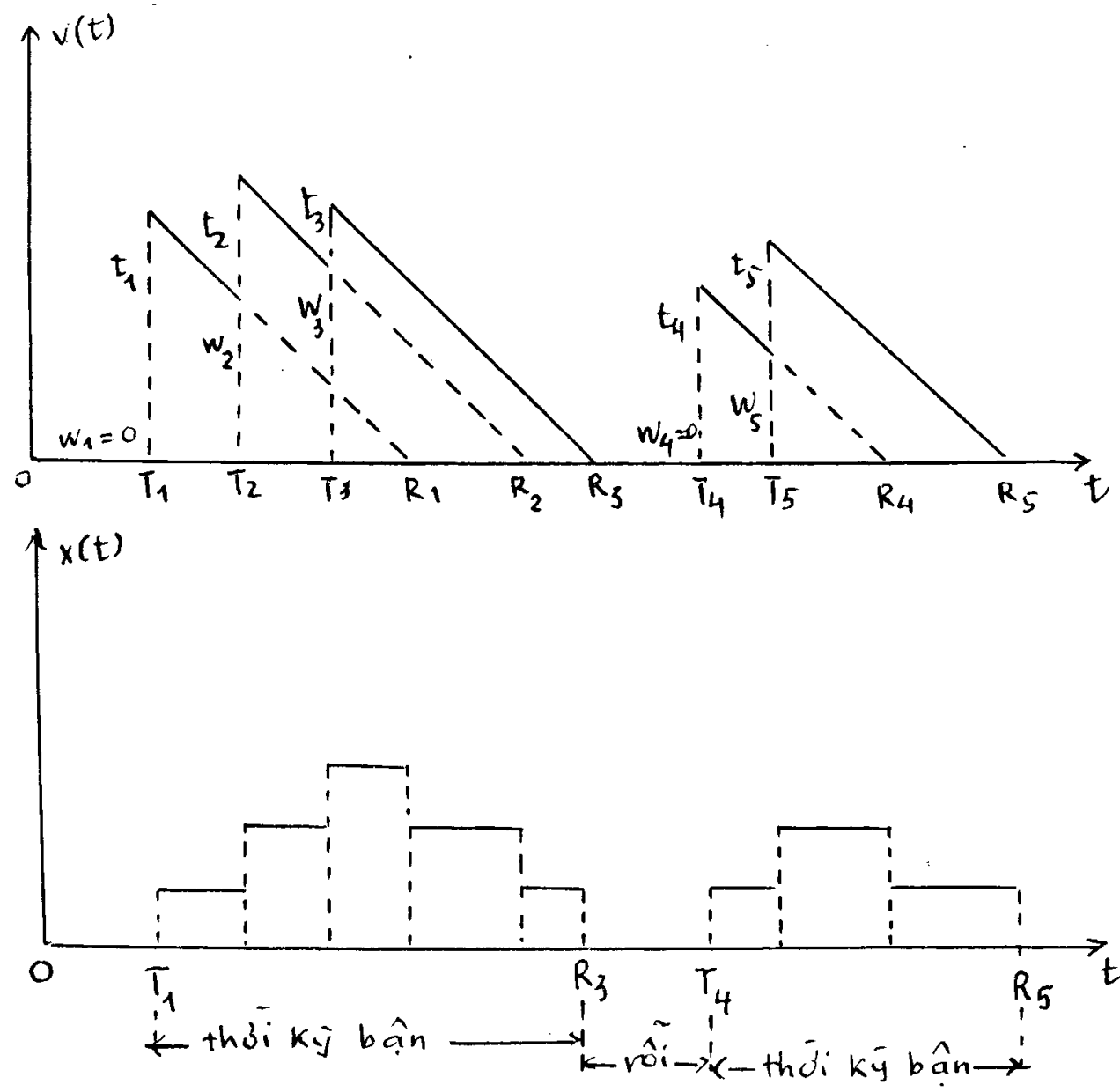

Hinh 1

Nój chung ngırời ta cần quan tâm đến tác động của hai yếu tố dần đển yểu tố thử ba vì đó là thước đo tính hiẹu quả cuia một hệ thống phục vụ.

Người $\mathrm{ta}$ chứng minh được rằng số yêu cầu của người đọc xuất hiện trong một khoảng thời gian $t$ là nộ̣t biển ngẫu nhiên $N$ tuân theo quy luạt Poisson và xác suất để có $N=n$ yêu cầu trong khoàng thời gian $t$ là

$$
P_{n}(t)=\left((\lambda t)^{n} e^{-\lambda t}\right) / n !
$$

trong đó

$$
\lambda=E(N) / t
$$

chính là số yêu cầu trung bình cửa người dọc trong một đơn vy thời gian. 
Nểu dòng yêu càu là dòng Poisson với trung bình $\lambda$ thì khoảng thø2wh i gian giữa hai yêu rảu liên tiép là biến ngẫu nhiên $\tau$ tuân theo quy luật phân phối mũ yới tham số $\lambda$, tức là hàm phân phối xỉx suất của nó có dạng

$$
F(t)= \begin{cases}0 & \text { nếu } t \leq 0 \\ 1-e^{-\lambda t} & \text { néu } t>0\end{cases}
$$

và

$$
E(\tau)=1 / \lambda
$$

là trung bình của khoảng thời gian giữa hai yêu cầu.

Còn với thời gian phục vụ một yêu cầu, người ta cũng chứng minh được rằng đó là một biến ngẫu nhiên $T$ tuân theo quy luật phân phói mũ với hàm phân phối là

$$
F(t)= \begin{cases}0 & \text { néu } t \leq 0 \\ 1-e^{-\mu t} & \text { néu } t>0 .\end{cases}
$$

Kìy vọng toán học của $T$ là

$$
E(T)=1 / \mu
$$

Do đó

$$
\mu=1 / E(T)
$$

chính là số yĉu câu trung bỉnh được phục vụ trong một dơn vị thời gian.

Dới với bài toán đặt ra, phương pháp mò phỏng đem lại nhiều lợi ich. Nhờ đó ta có thể phân tích đául giá được hiện trạng hoạt dộng của hệ thống pliục vụ, và xác định dược phương án phục vụ tốt l..rn.

\section{Mô phóng quá trình phuc vu người đọ.}

Trong [8] chúng tôi dã trỉnh bày các kết quà diều tra chọn mẫu và nghiên cúu hoạt dộng phục vụ ở plìng đọc của thư viện Khơ học kỹ thuật Trung ương, clúng tôi đã thu đırợc các kết quả sau day:

- Dòng yêu cầu của người dọc là dòng Poisson với trung bỉnh là $\lambda=0.08$ yêu cảu / phút. Khi đó khoàng thời gian giữa hai yêu câu là bićn ngẫu nhiên $\tau$ tuân theo quy luật phân phối inũ với trung bỉnh là $E(\tau)=1 / \lambda=1 / 0.08=1.25$ phút.

- Thời gian phục vụ $T$ tuân theo quy luạt phân phối mũ với trung bình là $E(T)=1 / \mu=6.72$ phút và $\mu=1 / 6.72=0.15$ là số yêu cảu trung bỉnh được phục vụ trong một phút.

Ta biết rằng nếu $\mathrm{x}$ là một biến ngẫu nliên liên tục, có hàm phân phối $F(x)$ và $u$ là một thể hị̣n của biến ngẫu nhién $U$ tuân theo quy luật phân phối đểu trên khoảng $[0,1]$ thì $x=F^{-1}(u)$ là một thể hic̣n mo phóng của bién ngẫu nhiên $X$.

Ở đay hàm phân phới của thời gian phục vụ $T$ có dạng

$$
F(l)= \begin{cases}0 & \text { với } t \leq 0 \\ 1-e^{-\mu t} & \text { với } t>0 .\end{cases}
$$


Hàm ngược của $F$ là

$$
F^{-1}(u)=-1 / \mu \cdot \ln (1-u)
$$

Do đó với số ngẫu nhiên $u_{1}$ thì giá trị mô phóng của thời gian phục vụ $T$ là

$$
T_{1}=-1 / \mu \cdot \ln u_{1}=6.72\left(-\ln {u_{1}}_{1}\right) .
$$

Tương tự vơoi số ngẫu nhiên $v_{1}$, giá trị mô phỏng của khoảng thời gian giữa hai yêu cầu liên tiếp $\tau$ là

$$
\tau_{1}=-1 / \lambda \ln v_{1}=12.5\left(-\ln v_{1}\right)
$$

Tìr mô hỉnh toán học của quá trỉnh phục vụ ngườ đọc trỉnh bày ở trên ta có thể xây dựng được thuật toán mô phóng quá trỉnh phục vụ người đọc ở thư viện Khoa học Kỹ thuật Trung ưng.

Già sử $T_{l}=0$ là thời điểm đến của yêu cẩu thứ nhất. Thời điểm đến của yêu câu tiếp theo được tính theo công thức

$$
T_{i}=T_{i-1}+\tau_{i}, i=1,2, \ldots
$$

trong đó $\tau_{i}$ là khoảng thời gian giữa hai yêu cầu liên tiếp thứ $i-1$ và $i$.

Tìr bảng số ngẫu nhiên, ta lấy ra một sổ ngẫu nhiên, chẳng hạn lấy từ dưới lên ta được số $v_{2}=0.69$. Khi đó khoảng thời gian đến giữa yêu cầu thứ nhất và thứ hai có giá trị mô phóng là $\tau_{2}=12.5(-\ln 0.69)=12.50 .37=4.64$ phút.

Lấy tiếp số ngẫu nhiên theo thứ tự từ trên xuống, ta được số $u_{1}=0.10$. Khi đó giá trị mô phóng thời gian phục vụ yêu cầu thứ nhất là $t_{1}=6.72(-\ln 10)=6.722 .3=15,47$ phút.

Thời gian đợi của yêu cầu thứ nhất là $w_{1}=0$ nên thời gian lưu và thời điểm yêu cẩu thứ nhất rời hệ là

$$
\begin{gathered}
d_{1}=w_{1}+t_{1}=0+15,47=15,47 \\
R_{1}=T_{1}+w_{1}+t_{1}=0+0+15,47=15,17
\end{gathered}
$$

Vì yêu cầu thứ hai đến vào thời điêm

$$
T_{2}=T_{1}+\tau_{2}=0+4,64=4,64
$$

trược thời điêm $R_{1}$ nên yêu cầu thứ hai phải xếp hàng và thời gian đợi là

$$
w_{2}=R_{1}-T_{2}=15,47-4,64=10,83
$$

Lấy số ngẫu nhiên tiếp theo $u_{2} \doteq 0.09$. Thời gian phục vụ yêu câu thử hai có giá trị mô phỏng là

$$
t_{2}=6.72(-\ln 0.09)=6.722 .41=16,18
$$

yêu cầu này rời hệ tại thời điểm

$$
R_{2}=T_{2}+w_{2}+t_{2}=4.64+10.83+16.18=31.65
$$


Thời gian lưu của yêu cầu thứ hai là

$$
d_{2}=w_{2}+t_{2}=10.83+16.18=27.01
$$

Lay sí ngẫu nhiên tiếp theo $v_{3}=0.07$, khoảng thời gian giữa yêu cầu thứ hai và thứ ba đượr mò phòng bời

$$
\tau_{3}=12.5(-\ln 0.07)=12.52 .66=33.24
$$

Thời đliền đén của yêu cầu thứ ba là

$$
T_{3}=T_{2}+\tau_{3}=4.64+33.24=37.88
$$

Ta nhạn thảy yêu cầu thứ ba đến sau lúc yêu cầu thứ hai rời hệ $\left(T_{3}>R_{2}\right)$ nên trong khoảng thời gian này hệ rỗi và thời gian rỗi trước lúc $\mathrm{y}-6 \mathrm{eu}$ cầu thứ ba đến hị̂ là

$$
\int_{3}=T_{3}-R_{2}=37.88-31.65=6.23
$$

Trước lúc đén hệ rỗi, nên yĉu cẩu thứ ba không phải dợi $\left(u_{3}=0\right)$. Thời gian phục vụ yêu cầu thứ ba, úng với số ngẫu nhiên $u_{3}=0.73$ dược mô phỏng bởi

$$
t_{3}=12.5(-\ln 0.73)=2.11
$$

Do đó yêu cầu thứ ba rời hộ vào thời điểm

$$
R_{3}=T_{3}+u_{3}+{ }^{\prime}{ }_{3}=37.88+0+2.11=39.99
$$

\begin{tabular}{|c|c|c|c|c|c|c|c|c|c|}
\hline $\begin{array}{l}\text { yêu } \\
\text { cầu }\end{array}$ & $\begin{array}{l}\text { Nó ng. } \\
\text { nhiçn }\end{array}$ & $\begin{array}{l}\text { K.t.g.gii } \\
2 \text { yêu cầ }\end{array}$ & $\begin{array}{l}\text { áThời d. } \\
\text { udén } T_{i}\end{array}$ & $\begin{array}{l}\text { Số ng. } \\
\text { nhiên }\end{array}$ & $\begin{array}{l}\text { T.g. ph. } \\
\text { vụ } t_{i}=\end{array}$ & $\begin{array}{l}\text { T.g d. } \\
w_{i}=\end{array}$ & $\begin{array}{l}\text { T.đr. } \\
\text { hệ } R_{i}\end{array}$ & $\begin{array}{l}\text { T.o } \mathrm{l} . \\
d_{i}=\end{array}$ & $\begin{array}{l}\text { T.g. rõi } \\
f_{i}=\end{array}$ \\
\hline$i$ & $r_{i}$ & $\begin{array}{l}\tau_{i}=12 . \\
\left(-\ln v_{i}\right)\end{array}$ & $\begin{array}{l}=T_{i-1} \\
+\tau_{i}\end{array}$ & $u_{i}$ & $\begin{array}{l}6.72 \ln ( \\
\left.-u_{i}\right)\end{array}$ & $\begin{array}{l}R_{i-1} \\
-T_{i}\end{array}$ & $\begin{array}{l}=T_{i} \\
+u_{i}+t_{i}\end{array}$ & $\begin{array}{l}u_{i} \\
+t_{i}\end{array}$ & $\begin{array}{l}T_{i} \\
-R_{i-1}\end{array}$ \\
\hline 1 & & & 0 & 0.10 & 15.47 & 0 & 15.47 & 15.47 & 0 \\
\hline 2 & 0.69 & 4.64 & 4.61 & 0.09 & 16.18 & 10.83 & 31.65 & 27.01 & 0 \\
\hline 3 & 0.07 & 33.24 & 37.88 & 0.73 & 2.11 & 0 & 39.99 & 2.11 & 6.23 \\
\hline 4 & 0.49 & 8.92 & 46.80 & 0.25 & 9.32 & 0 & 56.12 & 9.32 & 6.81 \\
\hline 5 & 0.41 & 11.14 & 57.94 & 0.33 & 7.45 & 0 & 65.39 & 7.45 & 1.82 \\
\hline 6 & 0.38 & 12.09 & 70.13 & 0.76 & 1.84 & 0 & 71.97 & 1.84 & 4.74 \\
\hline$\tau$ & 0.87 & 1.74 & 71.87 & 0.52 & 4.39 & 0.10 & 76.36 & 4.49 & 0 \\
\hline 8 & 0.63 & 5.78 & 77.65 & 0.01 & 30.95 & 0 & 108.60 & 30.95 & 1.29 \\
\hline 9 & 0.79 & 2.95 & 80.60 & 0.35 & 7.05 & 28.00 & 115.65 & 35.05 & 0 \\
\hline 10 & 0.19 & 20.76 & 101.36 & 0.86 & 1.01 & 14.29 & 116.66 & 15.30 & 0 \\
\hline$\sum$ & & & & & $S=95.77$ & $\mathrm{C}=53.22$ & $=\mathrm{Q}$ & $\mathrm{P}=14$ & $9 \mathrm{~L}=20$ \\
\hline
\end{tabular}

Thời gian lưu của yêu cầu thử ba là

$$
d_{3}=w_{3}+\iota_{3}=0+2.11=2.11
$$

Tiếp tục quá trình trên ta được bảng tính sau dây mô phỏng quá trình phục vụ cho $n=10$ người dọc liên tiếp

(f) (f) (g)

(f) 
Từ bảng trên ta có thể rúr ra kết luận sau đây đơi với quá trỉnh phục vụ người yêu cầu:

- Tông thời gian phục vụ là

- Tring thời gian rỗi của hệ là

$$
S=\sum t_{i}=95.77 \text { phut } .
$$

$$
L=\sum f_{i}=21.20 \text { phút. }
$$

- Thisi gian hoạt động của hệ là

$$
Q=L+S=95.77+20.89=116.66 \text { phút. }
$$

hay là

$$
Q=T_{10}+d_{10}=101.36+15.30=116.66 \text { phút. }
$$

- Tông thời gian đợi của người dọc là

$$
C=\sum w_{i}=53.22 \text { phút. }
$$

- Tông thời gian luru của ngırời dọc trên là

$$
P=S+C=95.77+53.22=148.99 \text { phút. }
$$

- Thiơi gian đợi trung bình là

$$
C / N=.53 .22 / 10=5.32 \text { phút. }
$$

- Thời gian lưu trung bình là

$$
P / N^{r}=118.99 / 10=14.89 \text { phút. }
$$

- Tỷ lệ giữa thời gian rỗi và thời gian hoạt dộng của hệ là

$$
L / Q=21.20 / 148.99=0.18
$$

Sau đây ta xày dựng một sơ dò khởi the hiện thuật toán mô phóng quá trỉnh phục người đọc đã được trìuh bày ở tren.

Ta sư dụng các ký hiệu sau day

$\tau$ - khoảng thời gian giữa hai yeu câu lien tiḉp.

$t$ - Thời gian phục vụ một yèu càu.

$w$ - Thời gian đợi của một yêu çiu.

$f$ - Thời gian rỏi của hộ trước lúc yêu càu dirn.

$T$ - Thời điềm dến clia một yêu cầu.

$R$ - Thời dièm yêu càu rời hẹ.

$S$ - Tởng thời gian phục vụ $N$ yeu cầu.

$C$ - Tồng thời gian dọi cuia $N$ yeu câu.

$P$ - Tồng thời gian lưu của $N$ yẹu câu.

$Q$ - 'Tông thờ gian hıạt dọng của hệ.

$L$ - Tông thời gian rồi của liệ.

Ta có các quan liệ sau

$$
P=S+C, Q=S+L \text {. }
$$


Dựa sào các điều đã trình bày trên đây chúng tôi đã viết rhương trình mô phỏng quá trình phục vụ người đọc ở một thư viện Khoa học và kỹ thuạt trung ương. Chương trình dược viết lằng ngôn ngữ BASIC. Iloạt động của chương trỉnh được tổ chức theo chế độ menu. Các tùy chọn là nhur sau:

1 - Yô phỏng số yêu cầu của người đọc.

2 - Yô phỏng thởi gian phục vụ.

3 - Vô phóng quá trinh phụe vụ.

4 - Ra khỏi chương trình.

Chương trình con 1 và 2 hoạt động như những chương trình độc lập, cung cấp cho ta những thông tin về dòng yêu cầu và thời gian phục vụ như: số yêu cầu trung bỉnh trong khoảng thời gian định trước, khoảng thời gian trung bỉnh giữa thời điểm đến của hai yêu cầu liên tiếp, thời gian trung bỉnh phục vụ một yêu cầu. Nhưng khi chương trinh con 3 hoạt động thì chương trỉnh con 1 và 2 cun cáp các tham số $\lambda$ và $\mu$ cho chương trình con 3 . Khi chạy chương trình này, ta chỉ cần đưa vào số yêu cầu $N$ càn mò phỏng.

Kết quả mô phỏng sẽ cung cấp cho ta những thơng tịn sau đây về quá trình pliục vụ $N$ yêu cầu của ngirìi đọ ở một thư vi ện:

- Tổng thời gian plụ̣c vụ.

- Tơng thời gian rỗi.

- Tồng thời gian săp hàng.

- Tông thời gian hoạt dộng của hệ.

- Tỳ lệ giữa thời gian rỗi và thời gian hoạt động của hệ.

- Trung bỉnh của thời gian dợi

- Trung bỉnh của trhời gian lưu.

Dó là những tham số đặc trưng cho một quá trình phục vụ.

Nhưng dữ liệu ban đầu cho chương trình này có hai loại:

- Phân phối thực nghiệm của sń yêu cầu.

- Phín phối thực nghiệm của thời gian phục vụ.

Khi tién hành cho chương trình chạy với những dữ liệu khác nhau, phân tích các kết quà mo phơng nhận được, ta có thẻ xác định được một hinh thức tổ chức phục vụ thích hợn theo yêu cầu đè ra.

\section{Tài liêu tham kháo}

1. Lee A.M., Les files d'attent, Theoric et application, Paris, Dunod, 1970.

2. Kaufmann A., Cours modern de calcul des probabilite's, Paris Albin Michel, 1965.

3. Bernad Grais., Méthodes statistiques, Paris, Dunod, 1981.

4. Leroudier J., La simulation à événements discrets, Surenes, Hommes et Technique, 1980.

5. Lebart Ludevic \& Fénelon Jean Pierre, Stastiques et informatique appliques, Paris, Dunod, 1975.

6. Cohen J.M., The single server queue, Amsterdam, North Holland Publ. Co. 1969. 
7. Boisgontier Jaques, Le basic et ses fichiers, Laguysur - marne cedex, P.S.I. 1984.

8. Doàn Phan Tân, Mô hỉnh hóa quá trỉnh phục vụ người đọc ở một thư viện KHKT, Tập san Công tác Thư viện Khoa học Kỹ thuật, (1988), nº 2, 14-24.

Abstract

Simulation of the process of service in a library

This article prescnts an application of queuing theory in order to establish a mathematical model for the process of service in a library or in information center.

On the basic of the model mentioned and applying the simulation method of Monte - Carlo with properties of random numbers, the author proposes an algorithm and a flowchart for writing a simulation program of this process. The input data of this program is composed:

- Distribution of the number of andience's requests.

- Distribution of the service time.

Affter running, this program will give the following information on service systcm: Total of service time, total of writing time, total of free time, average of service time, average of writing time...

Thus are sharacteristics features of the service system. 